\title{
Spectroscopy of short-lived radioactive molecules
}

https://doi.org/10.1038/s41586-020-2299-4

Received: 24 July 2019

Accepted: 13 March 2020

Published online: 27 May 2020

\section{Open access}

Check for updates

\author{
R. F. Garcia Ruiz, ${ }^{1, \bigotimes}$, R. Berger ${ }^{3 凶}$, J. Billowes ${ }^{4}$, C. L. Binnersley ${ }^{4}$, M. L. Bissell ${ }^{4}$, A. A. Breier ${ }^{5}$, \\ A. J. Brinson ${ }^{2}$, K. Chrysalidis' ${ }^{1}$, T. E. Cocolios ${ }^{6}$, B. S. Cooper ${ }^{4}$, K. T. Flanagan ${ }^{4,7}$, T. F. Giesen ${ }^{5}$, \\ R. P. de Groote ${ }^{8}$, S. Franchoo ${ }^{9}$, F. P. Gustafsson ${ }^{6}$, T. A. Isaev ${ }^{10}$, Á. Koszorús ${ }^{6}$, G. Neyens ${ }^{1,6}$, \\ H. A. Perrett ${ }^{4}$, C. M. Ricketts ${ }^{4}$, S. Rothe ${ }^{1}$, L. Schweikhard ${ }^{11}$, A. R. Vernon ${ }^{4}$, K. D. A. Wendt ${ }^{12}$, \\ F. Wienholtz ${ }^{1,11}$, S. G. Wilkins ${ }^{1} \&$ X. F. Yang ${ }^{13}$
}

\begin{abstract}
Molecular spectroscopy offers opportunities for the exploration of the fundamental laws of nature and the search for new particle physics beyond the standard model ${ }^{1-4}$. Radioactive molecules-in which one or more of the atoms possesses a radioactive nucleus-can contain heavy and deformed nuclei, offering high sensitivity for investigating parity- and time-reversal-violation effects ${ }^{5,6}$. Radium monofluoride, RaF, is of particular interest because it is predicted to have an electronic structure appropriate for laser cooling ${ }^{6}$, thus paving the way for its use in high-precision spectroscopic studies. Furthermore, the effects of symmetry-violating nuclear moments are strongly enhance $d^{5,7-9}$ in molecules containing octupole-deformed radium isotope ${ }^{10,11}$. However, the study of RaF has been impeded by the lack of stable isotopes of radium. Here we present an experimental approach to studying short-lived radioactive molecules, which allows us to measure molecules with lifetimes of just tens of milliseconds. Energetically low-lying electronic states were measured for different isotopically pure RaF molecules using collinear resonance ionisation at the ISOLDE ion-beam facility at CERN. Our results provide evidence of the existence of a suitable laser-cooling scheme for these molecules and represent a key step towards high-precision studies in these systems. Our findings will enable further studies of short-lived radioactive molecules for fundamental physics research.
\end{abstract}

Molecular systems provide a versatile physical environment in which to study the fundamental symmetries of nature and the interactions and properties of subatomic particles ${ }^{1,2,12,13}$. Among the four known fundamental forces, the weak force is the only one that is known to violate symmetry with respect to spatial inversion of all particle coordinates (known as parity violation), giving rise to various intriguing phenomena. Some of these parity-violating effects have been measured with high accuracy in atomic systems ${ }^{13-15}$, contributing to the most stringent low-energy tests of the Standard Model of particle physics. In certain molecules, effects resulting from both parity violation (P-odd) and time-reversal violation (T-odd) are considerably enhanced with respect to atomic systems $\mathrm{s}^{5,7,8,13,16}$, offering the means to explore unknown aspects of the fundamental laws of physics. The strengths of these interactions scale with atomic number, nuclear spin and nuclear deformation, and so molecular compounds of heavy radioactive nuclei are predicted to exhibit unprecedented sensitivity, with an enhancement of more than two orders of magnitude for effects that are P-odd or simultaneously P- and T-odd 5-8,17-20. $^{\text {. }}$
However, the experimental knowledge of radioactive molecules is scarce ${ }^{21}$, and quantum chemistry calculations often constitute the only source of information. Molecules possess complex quantum level structures, which renders spectroscopy of their structure considerably more challenging compared to atoms. Moreover, major additional experimental challenges must be overcome to study molecules containing heavy and deformed nuclei, which can have lifetimes of just a few milliseconds. These radioactive nuclei are very rare in nature or do not occur naturally and so must be produced artificially at specialized facilities, such as at the Isotope Separator On-line Device (ISOLDE) at CERN. Furthermore, molecules containing short-lived isotopes can only be produced in quantities smaller than $10^{-8} \mathrm{~g}$ (typically with rates of less than $10^{6}$ particles $\left.\mathrm{s}^{-1}\right)$. Thus, spectroscopic studies require particularly sensitive experimental techniques adapted to the properties of radioactive ion beams and the conditions present at radioactive-beam facilities. Here, we present an approach for performing laser spectroscopy of short-lived radioactive molecules, using the highly sensitive collinear resonance ionization method ${ }^{22}$. These results provide the first

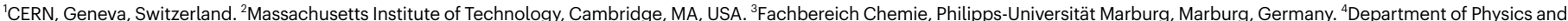
Astronomy, The University of Manchester, Manchester, UK. ${ }^{5}$ Laboratory for Astrophysics, Institute of Physics, University of Kassel, Kassel, Germany. ${ }^{6} \mathrm{KU}$ Leuven, Instituut voor Kern- en Stralingsfysica, Leuven, Belgium. ${ }^{7}$ Photon Science Institute, The University of Manchester, Manchester, UK. ${ }^{8}$ Department of Physics, University of Jyväskylä, Jyväskylä, Finland. ${ }^{9}$ Institut de Physique Nucleaire d'Orsay, Orsay, France. ${ }^{10} \mathrm{NRC}$ 'Kurchatov Institute'-PNPI, Gatchina, Russia. ${ }^{11}$ Institut für Physik, Universität Greifswald, Greifswald, Germany. ${ }^{12}$ Institut für Physik, Johannes Gutenberg-Universität Mainz, Mainz, Germany. ${ }^{13}$ School of Physics and State Key Laboratory of Nuclear Physics and Technology, Peking University, Beijing, China. ${ }^{\circledR e-m a i l: ~ r g a r c i a r @ m i t . e d u ; ~}$ robert.berger@uni-marburg.de 


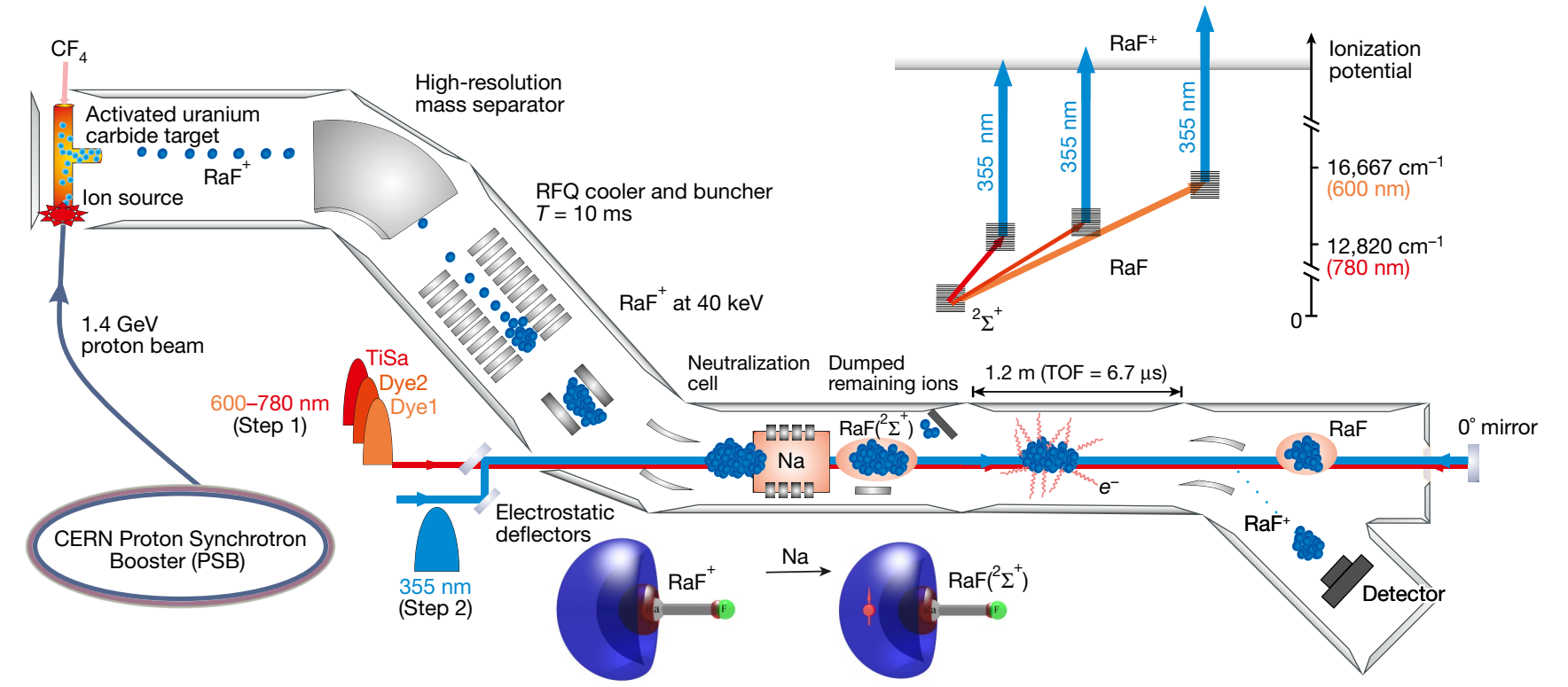

Fig. 1 Experimental scheme for the production and study of short-lived radioactive molecules. Radioactive radium isotopes were created by impinging 1.4-GeV protons from the CERN Proton Synchrotron Booster (PSB) on a uranium carbide $\left(\mathrm{UC}_{x}\right)$ target. Radium monofluoride cations $\left(\mathrm{RaF}^{+}\right)$were produced by passing tetrafluoromethane $\left(\mathrm{CF}_{4}\right)$ gas through the activated $\mathrm{UC}_{x}$ target at $1,300^{\circ} \mathrm{C}$. Molecular ions were extracted from the source, mass-selected and injected into a helium-filled RFQ trap, where they were accumulated for $10 \mathrm{~ms}$. Bunches of molecular ions were extracted and neutralized in flight by charge exchange with neutral sodium atoms. Neutral
RaF molecules were overlapped with different laser beams (step 1, TiSa, Dye1 and Dye2, and step 2, a 355-nm laser; see Methods section 'Laser setup') in a collinear geometry. Resonantly reionized molecules were deflected onto a particle detector. The resonance ionization scheme is shown at top right. At bottom, molecular orbitals are shown schematically. Nuclear positions within the molecules are coarsely indicated by a grey sphere $(\mathrm{Ra})$ and green sphere $(\mathrm{F})$, and the sigma bond between the atoms is indicated by the grey cylinders. Further details are provided in 'Experimental scheme'. spectroscopic information of $\mathrm{RaF}$, including isotopologues composed of radioactive isotopes with lifetimes as short as a few days. To our knowledge, this is the first laser spectroscopy study performed on a molecule containing a short-lived isotope. Moreover, this experimental scheme can be applied to study other radioactive molecules, even those composed of isotopes with lifetimes as short as a few tens of milliseconds.

Since the direct cooling of diatomic molecules with lasers ${ }^{23}$ was experimentally demonstrated ${ }^{24}$, there has been a wealth of studies on laser-cooling techniques and applications in molecular physics ${ }^{25-31}$. In contrast to other heavy-atom molecules, $\mathrm{RaF}$ is predicted to have highly closed excitation and re-emission optical cycles, which would make it ideal for laser cooling and trapping ${ }^{6}$. Moreover, owing to the recently discovered pear-shaped nuclear deformation of certain radium isotopes ${ }^{11}$, the interactions of the electrons with the P-odd nuclear anapole moment as well as with the P,T-odd nuclear Schiff and magnetic quadrupole moments are predicted to be enhanced by more than two orders of magnitude $e^{4,5,19,32}$. Hence, these molecules could provide a unique environment in which to measure these symmetry-violating nuclear moments.

\section{Experimental scheme}

Figure 1 shows a diagram of the experimental setup used to produce and study the RaF molecules. As a first step, radium isotopes were produced by diffusion out of an irradiated target (see Methods section 'Production of $\mathrm{RaF}$ molecules'). $\mathrm{RaF}^{+}$molecular ions were formed upon injection of $\mathrm{CF}_{4}$ gas into the target environment. The molecular ions were extracted from the ion source by applying an electrostatic field, and molecules containing one specific radium isotope were selected with a high-resolution magnetic mass separator $(\Delta m / m \approx 1 / 2,000)$. The ions were collisionally cooled in a radio-frequency quadrupole (RFQ) trap filled with helium gas at room temperature (about $300 \mathrm{~K}$ ).
After up to 10 ms of cooling time, bunches of $\mathrm{RaF}^{+}$with a 4- $\mu$ s temporal width were released and accelerated to 39,998(1) eV, before entering into the Collinear Resonance Ionisation Spectroscopy (CRIS) setup ${ }^{22,33,34}$. At the CRIS beam line, the ions were first neutralized in-flight by passing through a collision cell filled with a sodium vapour, inducing charge exchange according to the reaction $\mathrm{RaF}^{+}+\mathrm{Na} \rightarrow$ $\mathrm{RaF}+\mathrm{Na}^{+}$. As the ionization energy of $\mathrm{RaF}$ is estimated to be close to that of sodium $(5.14 \mathrm{eV})^{35}$, the neutralization reaction dominantly populates the $\mathrm{RaF} \mathrm{X}^{2} \Sigma^{+}$electronic ground state. Molecular pseudo-orbitals obtained from one-component open-shell (neutral) or closed-shell (ion) restricted Hartree-Fock calculations with an energy-consistent effective core potential on radium are shown schematically in Fig. 1 (bottom). The lowest unoccupied molecular orbital in $\mathrm{RaF}^{+}$, which is mainly of non-bonding character, becomes occupied by an unpaired electron (symbolized in Fig. 1 by a red sphere together with an arrow representing the electron spin) upon neutralization. This is shown schematically as an isodensity, with lobes in slightly transparent blue and transparent red indicating different relative phases of the single-electron wavefunction.

After the charge-exchange reaction, non-neutralized $\mathrm{RaF}^{+}$ions were deflected out of the beam, and the remaining bunch of neutral $\mathrm{RaF}$ molecules was overlapped in time and space by several (pulsed) laser beams in a collinear arrangement, along the ultrahigh-vacuum $\left(10^{-10} \mathrm{mbar}\right.$ ) interaction region of 1.2-m length. Laser pulses (step 1) of tunable wavelength were used to resonantly excite the transition of interest, and a high-power 355-nm laser pulse (step 2) was used to subsequently ionize the excited $\mathrm{RaF}$ molecules into $\mathrm{RaF}^{+}$(see Fig. 1, top). The resonantly ionized molecules were then separated from the non-ionized molecules by deflecting the ions onto a particle detector. When the excitation laser is on resonance with a transition in the molecule (step 1 in Fig. 1), the second laser pulse ionizes the molecule, producing a signal at the detector. Molecular excitation spectra were obtained by monitoring the ion counts as a function of the wavenumber of the first laser. 


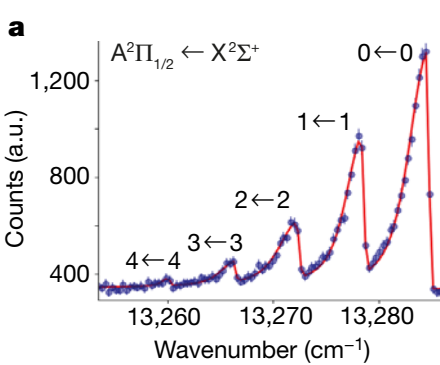

b

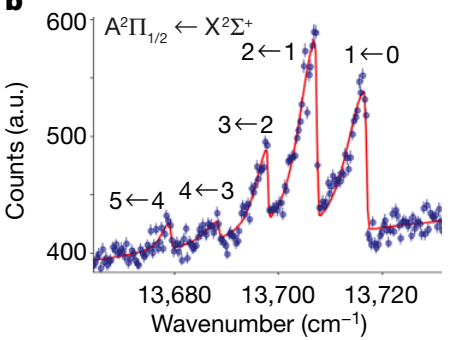

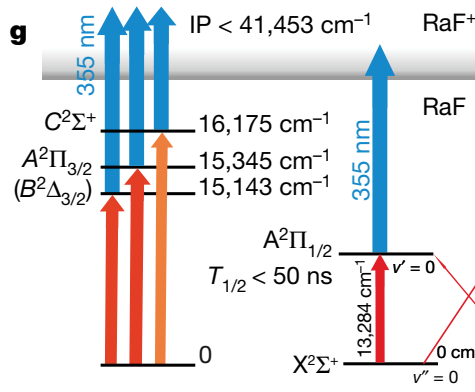

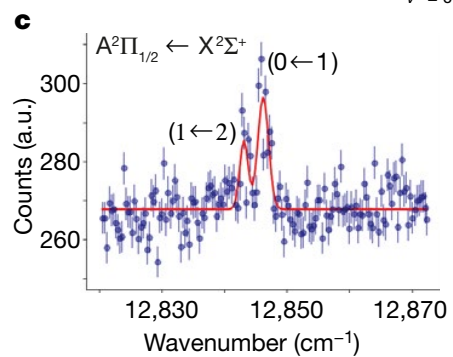

Fig. 2 | Examples of vibronic spectra measured for ${ }^{226}$ RaF. a-f, The counts on the particle detector were measured as a function of the laser wavenumber of the resonant step. A fixed wavelength $(355 \mathrm{~nm})$ was used for the ionization step. a, The observed peaks corresponding to the vibronic spectra of the $\Delta v=0$ band system of $v^{\prime \prime}=0,1,2,3,4$, scanned by the grating Ti:sapphire laser. b, c, The pulsed dye laser was used to scan electronic transitions in different wavelength ranges: the $\Delta v=+1$ band system of the $\mathrm{A}^{2} \Pi_{1 / 2} \leftarrow \mathrm{X}^{2} \Sigma^{+}$transition with $v^{\prime \prime}=0,1,2,3,4$ (b) and the $\left(v^{\prime}, v^{\prime \prime}\right)=(0,1)$ and $(1,2)$ band. $\mathbf{d}-\mathbf{f}$, The corresponding transitions to other electronic states: $\mathrm{A}^{2} \Pi_{3 / 2} \leftarrow \mathrm{X}^{2} \Sigma^{+}$(d), $\mathrm{B}^{2} \Delta_{3 / 2} \leftarrow \mathrm{X}^{2} \Sigma^{+}$(tentatively assigned; e) and $C^{2} \Sigma^{+} \leftarrow X^{2} \Sigma^{+}(\mathbf{f})$. The shape of the spectra is due to population distribution of different rotational states. The solid lines show the fit with skewed Voigt

Only theoretical predictions were available for the excitation energies of $\mathrm{RaF}$, and so finding the transition experimentally required scanning a large wavelength range $\left(>1,000 \mathrm{~cm}^{-1}\right)$. The prediction for the $A^{2} \Pi_{1 / 2}-X^{2} \Sigma^{+}(0,0)$ transition, for example, was $13,300 \mathrm{~cm}^{-1}$, with an accuracy estimated to be within $1,200 \mathrm{~cm}^{-1}$ (refs. ${ }^{6,32}$ ). Given the bandwidth of the commonly available lasers $\left(<0.3 \mathrm{~cm}^{-1}\right)$, the scan of such a large wavelength region on samples produced at rates below $10^{6} \mathrm{~mol}-$ ecules $\mathrm{s}^{-1}$ represented a major experimental challenge. To optimize the search of molecular transitions, three broadband lasers were scanned simultaneously and both collinearly and anti-collinearly (see Methods section 'Laser setup').

\section{Results}

The predicted region for the $A^{2} \Pi_{1 / 2} \leftarrow X^{2} \Sigma^{+}$transition was scanned at a speed of $0.06 \mathrm{~cm}^{-1} \mathrm{~s}^{-1}$, covering a range of $1,000 \mathrm{~cm}^{-1}$ in about $5 \mathrm{~h}$, using the six simultaneously applied scanning regions. After a few hours of scanning on a beam of ${ }^{226} \mathrm{RaF}$, a clear sequence of vibronic absorption signals was recorded. The measured spectrum assigned to the $\left(v^{\prime}, v^{\prime \prime}\right)$ vibrational transitions $(0,0),(1,1),(2,2),(3,3)$ and $(4,4)$ of the $A^{2} \Pi_{1 / 2}-$ $\mathrm{X}^{2} \Sigma^{+}$band system is shown in Fig. 2a. Weaker band structures, that were found at about $+440 \mathrm{~cm}^{-1}$ and $-440 \mathrm{~cm}^{-1}$ with respect to the $(0,0)$ band, were assigned to the $\Delta v= \pm 1$ transitions $\left(v^{\prime}, v^{\prime \prime}\right)=(1,0),(2,1),(3,2),(4,3)$, $(5,4)$ and $\left(v^{\prime}, v^{\prime \prime}\right)=(0,1),(1,2)$, respectively (Fig. 2b, c). The quantum number assignment for $\Delta v=-1$ is tentative, owing to the highly dense structure of overlapping vibronic bands.

In addition to the $A^{2} \Pi_{1 / 2}-X^{2} \Sigma^{+}$band system, we found spectroscopic signatures of electronic transitions to higher-lying states. Some examples of recorded spectra are shown in Fig. $2 d-f$, along with the energy-level scheme. We assign the observed transitions as follows: 1) The band system around $15,325 \mathrm{~cm}^{-1}$ (Fig. 2d) is attributed to the $\mathrm{A}^{2} \Pi_{3 / 2}-\mathrm{X}^{2} \Sigma^{+}$transition, owing to the complex rovibrational structure expected to arise from the intense satellites that are possible in these transitions. Because the bands are comparatively strong, they are assigned to the $\Delta v=0$ band system. Although the individual assignments to vibrational transitions must be considered to be tentative, as per the congested structure of the Franck-Condon profile, the $\Delta v=0$ assignment is substantiated because no additional structure was located within a relative range of -400 to $+400 \mathrm{~cm}^{-1}$. The band system located around $15,143 \mathrm{~cm}^{-1}$ (Fig. 2e) is tentatively assigned to the $\mathrm{B}^{2} \Delta_{3 / 2}-\mathrm{X}^{2} \Sigma^{+}$transition by virtue of the good agreement with the computed excitation energies to the $\Omega=3 / 2$ state of mixed $\Delta / \Pi$ character ${ }^{6,32}$. This mixing provides intensity to the one-photon transition from a $\Sigma$ state into the $\Delta$ manifold. The computed Born-Oppenheimer potentials for this $\Omega=3 / 2$ state and the electronic ground state are, however, highly parallel, which would suggest a sparser Franck-Condon profile than was observed experimentally. However, we note that the related $\mathrm{B}^{2} \Delta_{3 / 2}-\mathrm{X}^{2} \Sigma^{+}$transition in $\mathrm{BaH}$ and $\mathrm{BaD}$ was reported to have a perturbed character owing to mixing between electronic levels ${ }^{36}$. Thus, in the present case, a vibrational profile that is richer than expected from adiabatic potentials cannot be ruled out a priori. The band system with origin at $16,175 \mathrm{~cm}^{-1}$ (Fig. $2 \mathrm{f}$ ) is assigned to the $\mathrm{C}^{2} \Sigma^{+}-\mathrm{X}^{2} \Sigma^{+}$transition on the basis of the observed Franck-Condon profile, which is in good agreement with the computed harmonic vibrational energy spacings as well as the expected intensity distribution, and is in a wavenumber region that is only slightly lower than predicted ${ }^{6,32}$. All measured and assigned vibronic bands of the four electronic transitions are listed in Table 1.

The measured $\mathrm{A}^{2} \Pi_{1 / 2}-\mathrm{X}^{2} \Sigma^{+}(0,0)$ band centre, $\tilde{\mathcal{T}}_{\mathrm{e}}=13,287.8(1) \mathrm{cm}^{-1}$ is in excellent agreement with the ab initio calculated value of $13,300(1,200) \mathrm{cm}^{-1}\left(\right.$ ref. $\left.^{32}\right)$. In accordance with theoretical predictions ${ }^{6}$, we found vibronic transitions with $\Delta v=0$ to be much stronger than those with $\Delta v= \pm 1$. For most of the measurements, the power density used for the resonant step was $100(5) \mu \mathrm{J} \mathrm{cm}^{-2}$ per pulse, as measured 
Table 1 | Measured vibronic transitions of ${ }^{226} \operatorname{RaF}$ from the $X^{2} \Sigma^{+}$ electronic ground state to the excited $A^{2} \Pi$ and $B^{2} \Delta$ states

\begin{tabular}{|c|c|c|}
\hline Transition & $v^{\prime} \leftarrow v^{\prime \prime}$ & $\Delta \tilde{v}\left(\mathrm{~cm}^{-1}\right)$ \\
\hline \multirow[t]{12}{*}{$\mathrm{A}^{2} \Pi_{1 / 2} \leftarrow \mathrm{X}^{2} \Sigma^{+}$} & $0-0$ & $13,284.7(5)$ \\
\hline & $1-1$ & $13,278.5(5)$ \\
\hline & $2-2$ & $13,272.4(5)$ \\
\hline & $3-3$ & $13,266.4(10)$ \\
\hline & $4-4$ & $13,260.2(10)$ \\
\hline & $1-0$ & $13,716.9(5)$ \\
\hline & $2-1$ & $13,707.4(5)$ \\
\hline & $3-2$ & $13,698.0(5)$ \\
\hline & $4-3$ & $13,688.6(10)$ \\
\hline & $5-4$ & $13,679.4(10)$ \\
\hline & $(0-1)$ & $12,846.3(10)$ \\
\hline & $(1-2)$ & $12,843.1(10)$ \\
\hline \multirow[t]{4}{*}{$\left(\mathrm{B}^{2} \Delta_{3 / 2} \leftarrow \mathrm{X}^{2} \Sigma^{+}\right)$} & $0-0$ & $15,142.7(5)$ \\
\hline & $1-1$ & $15,132.8(10)$ \\
\hline & $2-2$ & $15,123.0(10)$ \\
\hline & $3-3$ & $15,113.2(10)$ \\
\hline \multirow[t]{3}{*}{$\mathrm{A}^{2} \Pi_{3 / 2} \leftarrow \mathrm{X}^{2} \Sigma^{+}$} & $(0-0)$ & $15,344.6(50)$ \\
\hline & $(1-1)$ & $15,325.0(80)$ \\
\hline & $(2-2)$ & $15,309.4(100)$ \\
\hline \multirow[t]{4}{*}{$\mathrm{C}^{2} \Sigma^{+} \leftarrow \mathrm{X}^{2} \Sigma^{+}$} & $0-0$ & $16,175.2(5)$ \\
\hline & $1-1$ & $16,164.2(5)$ \\
\hline & $2-2$ & $16,153.4(5)$ \\
\hline & $3-3$ & $16,142.4(10)$ \\
\hline
\end{tabular}

The values indicate the band head positions.

Combined statistical and systematic uncertainties are included in parentheses.

The $\mathrm{B}^{2} \Delta_{3 / 2} \leftarrow \mathrm{X}^{2} \Sigma^{+}$assignment is tentative.

at the entry window of the beam line. Reducing the power by $50 \%$ did not reduce the resonant ionization rate, indicating that these transitions were measured well above saturation. The much weaker vibrational transitions with $\Delta v= \pm 1$ were scanned with a pulsed dye laser of $500(5) \mu \mathrm{J} \mathrm{cm}^{-2}$ power density per pulse (bandwidth of $0.1 \mathrm{~cm}^{-1}$ ). The $\Delta v= \pm 1$ transitions were measured well above saturation and with laser beams of different characteristics, and so a precise estimation of the Franck-Condon factors could not be obtained. Instead, a lower limit of 0.97 for the peak intensity ratio $I(0,0) / I(0,1)$ was derived, indicating highly diagonal Franck-Condon factors, an essential property for laser cooling'.

By measuring the resonant ionization rate for different time delays between the excitation and ionization laser pulses, we obtained an upper limit for the lifetime of the excited state ${ }^{2} \Pi_{1 / 2}\left(v^{\prime}=0\right): T_{1 / 2} \leq 50 \mathrm{~ns}$. The measurements were performed with the wavenumber of the resonant laser fixed at the resonance value of the transition $\left(v^{\prime}, v^{\prime \prime}\right)=(0,0)$. The resonant ionization rate dropped by more than $70 \%$ for delays above $50 \mathrm{~ns}$. This short lifetime corresponds to a large spontaneous decay rate $\left(>2 \times 10^{7} \mathrm{~s}^{-1}\right)$, which would allow for the application of strong optical forces for laser cooling. An additional concern for the suitability of laser cooling is related to the existence of metastable states lying energetically below the ${ }^{2} \Pi_{1 / 2}$ level, which could prevent the application of a closed optical-cooling loop, a major problem encountered for $\mathrm{BaF}$ ( $\mathrm{ref}^{6}$.). In contrast to $\mathrm{BaF}$, all other predicted electronic states $\left({ }^{2} \Pi_{3 / 2},{ }^{2} \Delta_{3 / 2}\right.$ and $\left.{ }^{2} \Sigma\right)$ in RaF were found to be energetically above the ${ }^{2} \Pi_{1 / 2}$ state, indicating that its electronic structure will allow for efficient optical-cooling cycles.

From combination differences of energetically low-lying vibronic transitions in the band system $A^{2} \Pi_{1 / 2}-X^{2} \Sigma^{+}$, we have derived
Table $2 \mid{ }^{226}$ RaF Morse potential parameters for $\mathrm{X}^{2} \Sigma^{+}$ electronic ground and $A^{2} \Pi_{1 / 2}$ excited states

\begin{tabular}{lll}
\hline Parameter & $\tilde{\omega}_{\mathbf{e}}\left(\mathbf{c m}^{-1}\right)$ & $\tilde{\mathcal{D}}_{\mathbf{e}}\left(\times 10^{-4} \mathbf{c m}^{-1}\right)$ \\
\hline $\mathrm{X}^{2} \Sigma^{+}$ & $441.8(1)$ & $2.92(5)$ \\
\hline $\mathrm{A}^{2} \Pi_{1 / 2}$ & $435.5(1)$ & $2.90(3)$ \\
\hline
\end{tabular}

experimental values for the harmonic frequency, $\tilde{\omega}_{\mathrm{e}}$, and the dissociation energies, $\tilde{\mathcal{D}}_{\mathrm{e}}$, using a Morse potential approximation. Results are given in Table 2, and further details of the analysis can be found in Methods section 'Spectroscopic analysis'.

Furthermore, we measured the $A^{2} \Pi_{1 / 2} \leftarrow X^{2} \Sigma^{+}$vibronic spectra of ${ }^{226} \mathrm{RaF}$ and the short-lived isotopologues ${ }^{223} \mathrm{RaF},{ }^{224} \mathrm{RaF},{ }^{225} \mathrm{RaF}$, and ${ }^{228} \mathrm{RaF}$ (Fig. 3). All vibrational transitions were clearly observed, including those of the molecule with the shortest-lived radium isotope studied, ${ }^{224} \mathrm{RaF}\left(T_{1 / 2}=3.6 \mathrm{~d}\right)$. An on-line irradiation of the target material will enable the study of molecules containing isotopes with lifetimes as short as a few tens of milliseconds. The main limitation is dictated by the release from the target and the time spent in the RFQ trap $(>5 \mathrm{~ms})$. Future high-resolution measurements will enable studies of nuclear structure changes resulting from different isotopes and nuclear spins.

\section{Conclusions and future perspectives}

In summary, this Article presents an experimental approach for performing laser spectroscopy studies of molecules containing radioactive nuclei, which are typically produced at rates lower than $10^{6} \mathrm{~mol}-$ ecules $\mathrm{s}^{-1}$. Our results have established the energetically low-lying electronic structure of RaF, providing experimental evidence for the suitability of this diatomic molecule in a laser-cooling scheme. These findings are a pivotal step towards precision measurements in this system, which are expected to provide a highly sensitive environment for the exploration of physics beyond the Standard Model of particle physics.

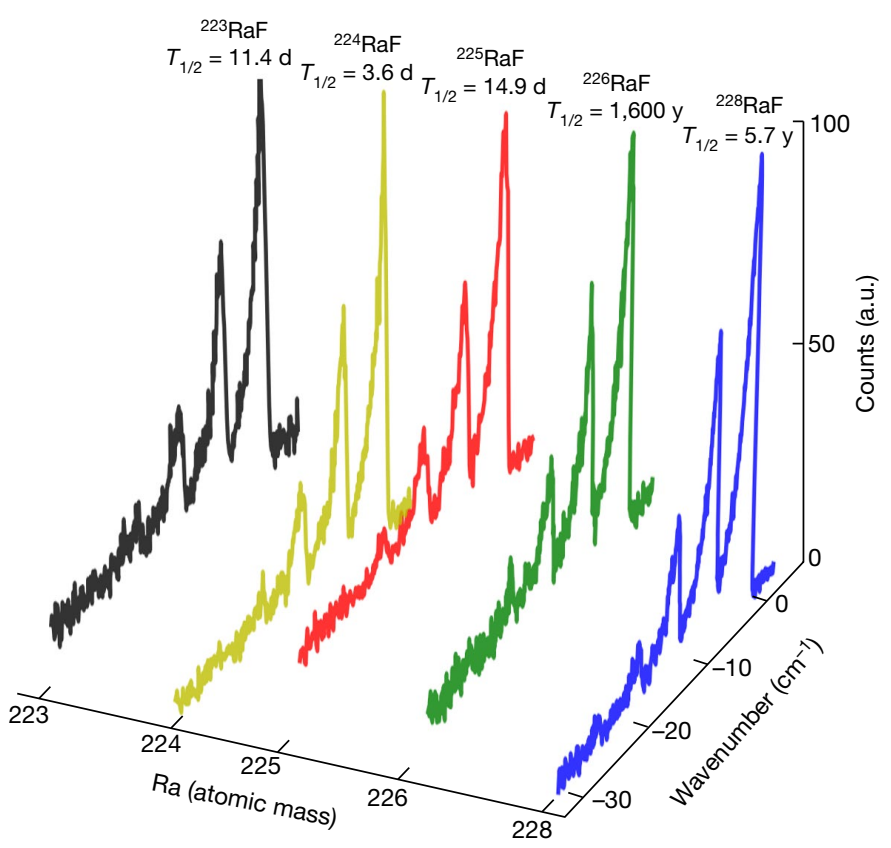

Fig. 3 | Vibronic spectra measured for different isotopologues of RaF. Measured vibronic absorption spectra for the $\mathrm{A}^{2} \Pi_{1 / 2} \leftarrow \mathrm{X}^{2} \Sigma^{+}$transition are shown for the isotopologues ${ }^{223} \mathrm{RaF}^{224} \mathrm{RaF}^{225} \mathrm{RaF},{ }^{226} \mathrm{RaF}$ and ${ }^{228} \mathrm{RaF}$. Wavenumber values are relative to the transition $(0,0)$ of ${ }^{226} \mathrm{RaF}$. 
Our experimental scheme can also be used to perform laser spectroscopy of a wide variety of neutral molecules and molecular ions, including those composed of isotopes with lifetimes of a few tens of milliseconds. Radioactive molecules can be precisely tailored to enhance their sensitivity to parity-and time-reversal-violating effects by introducing heavy and octupole-deformed nuclei. Moreover, by systematically replacing their constituent nuclei with different isotopes of the same element, both nuclear-spin-independent and nuclear-spin-dependent effects can be comprehensively studied. In addition, the present technique is applicable to other molecules of interest in studies of fundamental physics that are as yet experimentally unexplored, such as $\mathrm{RaOH}\left(\right.$ ref. $^{37}$ ), $\mathrm{RaO}$ (ref. ${ }^{18}$ ), $\mathrm{RaH}$ (ref. ${ }^{17}$ ), AcF (ref. ${ }^{38}$ ) and ${ }^{229}$ ThO (ref. ${ }^{5}$.

In addition to the impact of our findings on quantum chemistry, nuclear structure and fundamental physics research, the ability to produce, mass-select and spectroscopically study short-lived radioactive molecules is of importance to other fields of research such as radiochemistry ${ }^{21}$ and astrophysics ${ }^{39,40}$. Laboratory measurements of the spectra of radioactive molecules of astrophysical interest will allow their unambiguous identification in future astronomical observations. Furthermore, the possibility of performing spectroscopy on fast molecular beams will enable sub-Doppler spectroscopy to be performed even on molecules created at high temperatures ( $>600 \mathrm{~K})$. Thus, we expect our results will motivate further avenues of research at the increasingly capable radioactive-ion-beam facilities around the world.

\section{Online content}

Any methods, additional references, Nature Research reporting summaries, source data, extended data, supplementary information, acknowledgements, peer review information; details of author contributions and competing interests; and statements of data and code availability are available at https://doi.org/10.1038/s41586-020-2299-4.

1. Altuntaş, E. et al. Demonstration of a sensitive method to measure nuclear-spin-dependent parity violation. Phys. Rev. Lett. 120, 142501 (2018).

2. ACME Collaboration. Improved limit on the electric dipole moment of the electron. Nature 562, 355-360 (2018).

3. Berger, R. \& Stohner, J. Parity violation. WIREs Comput. Mol. Sci. 9, e1396 (2019).

4. Flambaum, V. V., DeMille, D. \& Kozlov, M. G. Time-reversal symmetry violation in molecules induced by nuclear magnetic quadrupole moments. Phys. Rev. Lett. 113, 103003 (2014)

5. Flambaum, V. V. Enhanced nuclear Schiff moment and time reversal violation in ${ }^{229}$ Th-containing molecules. Phys. Rev. C 99, 035501 (2019).

6. Isaev, T. A., Hoekstra, S. \& Berger, R. Laser-cooled RaF as a promising candidate to measure molecular parity violation. Phys. Rev. A 82, 052521 (2010).

7. Auerbach, N., Flambaum, V. V. \& Spevak, V. Collective T- and P-odd electromagnetic moments in nuclei with octupole deformations. Phys. Rev. Lett. 76, 4316-4319 (1996).

8. Kudashov, A. D. et al. Ab initio study of radium monofluoride ( $\mathrm{RaF}$ ) as a candidate to search for parity- and time-and-parity-violation effects. Phys. Rev. A 90, 052513 (2014).

9. Parker, R. H. et al. First measurement of the atomic electric dipole moment of ${ }^{225} \mathrm{Ra}$. Phys. Rev. Lett. 114, 233002 (2015).

10. Butler, P. A. et al. Evolution of octupole deformation in radium nuclei from Coulomb excitation of radioactive ${ }^{222} \mathrm{Ra}$ and ${ }^{228} \mathrm{Ra}$ beams. Phys. Rev. Lett. 124, 042503 (2020).

11. Gaffney, L. et al. Studies of pear-shaped nuclei using accelerated radioactive beams. Nature 497, 199-204 (2013).

12. Cairncross, W. B. et al. Precision measurement of the electron's electric dipole moment using trapped molecular ions. Phys. Rev. Lett. 119, 153001 (2017).
13. Safronova, M. S. et al. Search for new physics with atoms and molecules. Rev. Mod. Phys. 90, 025008 (2018).

14. Antypas, D. et al. Isotopic variation of parity violation in atomic ytterbium. Nat. Phys. 15, 120-123 (2019).

15. Wood, C. S. et al. Measurement of parity nonconservation and an anapole moment in cesium. Science 275, 1759-1763 (1997)

16. Sandars, P. G. H. Measurability of the proton electric dipole moment. Phys. Rev. Lett. 19, 1396-1398 (1967).

17. Fazil, N. M., Prasannaa, V. S., Latha, K. V. P., Abe, M. \& Das, B. P. RaH as a potential candidate for electron electric-dipole-moment searches. Phys. Rev. A 99, 052502 (2019).

18. Flambaum, V. V. Electric dipole moments of actinide atoms and RaO molecule. Phys. Rev. A 77, 024501 (2008).

19. Gaul, K., Marquardt, S., Isaev, T. A. \& Berger, R. Systematic study of relativistic and chemical enhancements of P,T-odd effects in polar diatomic radicals. Phys. Rev. A 99, 032509 (2019).

20. Sasmal, S., Pathak, H., Nayak, M. K., Vaval, N. \& Pal, S. Relativistic coupled-cluster study of $\mathrm{RaF}$ as a candidate for the parity- and time-reversal-violating interaction. Phys. Rev. A 93, 062506 (2016).

21. Formanuik, A. et al. Actinide covalency measured by pulsed electron paramagnetic resonance spectroscopy. Nat. Chem. 9, 578-583 (2017).

22. Flanagan, K. T. et al. Collinear resonance ionization spectroscopy of neutron-deficient francium isotopes. Phys. Rev. Lett. 111, 212501 (2013).

23. Di Rosa, M. D. Laser-cooling molecules. Eur. Phys. J. D 31, 395-402 (2004).

24. Shuman, E. S. et al. Laser cooling of a diatomic molecule. Nature 467, 820-823 (2010).

25. Anderegg, L. et al. Laser cooling of optically trapped molecules. Nat. Phys. 14, 890-893 (2018).

26. Barry, J. F. et al. Magneto-optical trapping of a diatomic molecule. Nature 512, 286-289 (2014).

27. Hudson, J. J. et al. Improved measurement of the shape of the electron. Nature 473, 493-496 (2011).

28. Isaev, T. A. \& Berger, R. Polyatomic candidates for cooling of molecules with lasers from simple theoretical concepts. Phys. Rev. Lett. 116, 063006 (2016).

29. Isaev, T. A. \& Berger, R. Towards ultracold chiral molecules. Chimia 72, 375-378 (2018)

30. Lim, J. et al. Laser cooled YbF molecules for measuring the electron's electric dipole moment. Phys. Rev. Lett. 120, 123201 (2018).

31. Truppe, S. et al. Molecules cooled below the Doppler limit. Nat. Phys. 13, 1173-1176 (2017).

32. Isaev, T. A. \& Berger, R. Lasercooled radium monofluoride: a molecular all-in-one probe for new physics. Preprint at http://arxiv.org/abs/1302.5682 (2013).

33. de Groote, R. P. et al. Use of a continuous wave laser and Pockels cell for sensitive high-resolution collinear resonance ionization spectroscopy. Phys. Rev. Lett. 115, 132501 (2015).

34. Garcia Ruiz, R. F. et al. High-precision multiphoton ionization of accelerated laser-ablated species. Phys. Rev. X 8, 041005 (2018).

35. Isaev, T. A. et al. Ion neutralisation mass-spectrometry route to radium monofluoride. Preprint at http://arxiv.org/abs/1310.1511 (2013).

36. Bernard, A. et al. The $5 d$ states of barium hydride; BaH and BaD. Mol. Phys. 67, 1-18 (1989).

37. Isaev, T. A., Zaitsevskii, A. V. \& Eliav, E. Laser-coolable polyatomic molecules with heavy nuclei. J. Phys. B 50, 225101 (2017).

38. Flambaum, V. V. \& Dzuba, V. A. Electric dipole moments of atoms and molecules produced by enhanced nuclear Schiff moments. Phys. Rev. A 101, 042504 (2020).

39. Breier, A. A. et al. Lowest bending mode of ${ }^{13} \mathrm{C}$-substituted $\mathrm{C}_{3}$ and an experimentally derived structure. J. Chem. Phys. 145, 234302 (2016).

40. Kamiński, T. et al. Astronomical detection of radioactive molecule ${ }^{26} \mathrm{AlF}$ in the remnant of an ancient explosion. New Astron. 2, 778-783 (2018).

Publisher's note Springer Nature remains neutral with regard to jurisdictional claims in published maps and institutional affiliations.

Open Access This article is licensed under a Creative Commons Attribution 4.0 International License, which permits use, sharing, adaptation, distribution and reproduction in any medium or format, as long as you give appropriate credit to the original author(s) and the source, provide a link to the Creative Commons license, and indicate if changes were made. The images or other third party material in this article are included in the article's Creative Commons license, unless indicated otherwise in a credit line to the material. If material is not included in the article's Creative Commons license and your intended use is not permitted by statutory regulation or exceeds the permitted use, you will need to obtain permission directly from the copyright holder. To view a copy of this license, visit http://creativecommons.org/licenses/by/4.0/.

(c) The Author(s) 2020 


\section{Methods}

\section{Production of RaF molecules}

Ra isotopes were produced $33 \mathrm{~d}$ before the laser-spectroscopy measurements by impinging $1.4 \mathrm{GeV}$ protons on the cold $\mathrm{UC}_{x}$ target material. The target was exposed to pulses of $10^{13}$ protons per pulse over a period of $2 \mathrm{~d}$. After irradiation with a total of $8 \times 10^{17}$ protons, the target was kept in a sealed chamber filled with Ar gas. After day 33, the target was connected to the High-Resolution Separator (HRS) front-end at ISOLDE. FLUKA ${ }^{41}$ simulations predicted $2 \times 10^{13}$ atoms of ${ }^{226} \mathrm{Ra}$ in the target material $\left(7.5 \times 10^{-9} \mathrm{~g}\right)$, following proton irradiation of a cold target. The target was pumped down to pressures below $10^{-5} \mathrm{mbar}$, and the target holder and ion source were gradually heated up to about $1,300^{\circ} \mathrm{C}$, in order for the Ra isotopes to diffuse towards the surface of the target material. A leak valve attached to the target was used to inject $\mathrm{CF}_{4}$ into the target environment. $\mathrm{The} \mathrm{CF}_{4}$ molecules dissociate and react with atoms and molecules on the target surface until an equilibrium is reached. $\mathrm{RaF}$ molecules were formed by reactive collisions of $\mathrm{CF}_{4}$ molecules with $\mathrm{Ra}$ atoms present inside the irradiated target material.

According to thermodynamic equilibrium calculations ${ }^{42}, \mathrm{RaF}_{2}$ or $\mathrm{RaF}$ are expected to form, depending on the local temperature. Within the temperature gradient between the target $\left(1,300^{\circ} \mathrm{C}\right)$ and the ion source $\left(2,000^{\circ} \mathrm{C}\right), \mathrm{RaF}_{2}$ fully reacts to form $\mathrm{RaF}$. A measured ratio of the ion-beam intensity of $\mathrm{Ra}^{+}$to $\mathrm{RaF}^{+}$of less than 0.05 indicates that more than $95 \%$ of the Ra isotopes released from the target material are converted and extracted as molecules.

$\mathrm{The}^{226} \mathrm{RaF}^{+}(A=245)$ beam extracted from the ISOLDE target unit was sent to the ISOLTRAP setup ${ }^{43}$, where the molecular ions were captured, cooled and bunched by a different RFQ trap and subsequently analysed using a multi-reflection time-of-flight mass spectrometer ${ }^{44}$. A measured mass spectrum is shown in Extended Data Fig. 1. After 1,000 revolutions in the device, a mass resolving power $(R=m / \Delta m)$ of $1.7 \times 10^{5}$ was achieved, which allowed the isobaric beam composition to be analysed. The only mass peak detected was identified as the signal of ${ }^{226} \mathrm{Ra}^{19} \mathrm{~F}^{+}$, confirming the purity of the beam from ISOLDE.

The intensity of $\mathrm{RaF}^{+}$molecules depends strongly on the target and ion source temperature. For a target temperature of $1,300^{\circ} \mathrm{C}$, a mean value of $2 \times 10^{7}$ molecules $\mathrm{s}^{-1}$ of $^{226} \mathrm{RaF}^{+}$was measured after the mass separator. Depending on the molecular mass and beam intensity, the transmission efficiency through the RFQ trap varied from $15 \%$ to $30 \%$. The ion-beam transmission from the ion trap to the interaction region was measured to be $25(5) \%$. The charge exchange cell vapour was heated to produce a measured neutralization rate of $30(5) \%$. Thus, we estimate that on average $5 \times 10^{4}$ neutral ${ }^{226} \mathrm{RaF}$ molecules $\mathrm{s}^{-1}$ were delivered to be resonantly excited. From the analysis of the measured spectra it was concluded that the neutral molecules populate the low-lying vibrational states $v=0,1,2,3,4$ following a relative population of 0.47:0.29:0.13:0.05:0.03. Resonantly ionized molecules with rates of the order of $10^{3}$ counts s$^{-1}$ at the peak of the $0 \leftarrow 0$ transition were measured at the particle detector. Future production of $\mathrm{RaF}^{+}$ molecular rates of the order of $10^{9}-10^{10}$ molecules $\mathrm{s}^{-1}$ is feasible using active proton irradiation ${ }^{45}$.

\section{Laser setup}

The resonance ionization schemes used for the study of $\mathrm{RaF}$ molecules are shown in Fig. 1. Three different laser systems were prepared to cover the scanning range from $12,800 \mathrm{~cm}^{-1}$ to $\left.13,800 \mathrm{~cm}^{-1}: 1\right)$ A dye-laser system (Dye1; Spectrolase 4000, Spectron) provided pulses of $100(5) \mu \mathrm{J}$ with a linewidth of $\left.10 \mathrm{GHz}\left(0.3 \mathrm{~cm}^{-1}\right) .2\right)$ A dye laser (Dye2; Cobra, Sirah) with a narrower linewidth of $2.5 \mathrm{GHz}\left(0.09 \mathrm{~cm}^{-1}\right)$ produced pulses of similar energy. The lasers were loaded with either Styryl 8 or DCM dyes to provide wavenumber ranges $12,800-14,000 \mathrm{~cm}^{-1}$ and $15,150-$ $16,600 \mathrm{~cm}^{-1}$, respectively. Both dye lasers were pumped by $532-\mathrm{nm}$ pulses at $100 \mathrm{~Hz}$, obtained from two different heads of a twin-head Nd:YAG laser (LPY 60150-100 PIV, Litron).3) A grating Ti:sapphire laser system with a linewidth of $2 \mathrm{GHz}\left(0.07 \mathrm{~cm}^{-1}\right)$ produced pulses of $20(1) \mu \mathrm{J}$, pumped by $532-\mathrm{nm}$ pulses at $1 \mathrm{kHz}$ from a Nd:YAG laser (LDP-100MQ, LEE Laser). The non-resonant ionization step was obtained by $355-\mathrm{nm}$ pulses of $30 \mathrm{~mJ}$ at $100 \mathrm{~Hz}$, produced by the third-harmonic output of a high-power Nd:YAG laser (TRLi 250-100, Litron).

The release of the ion bunch was synchronized with the laser pulses by triggering the flash-lamps and Q-switch of the pulsed lasers with a digital delay pulse generator (Quantum Composers 9528).

The dye-laser wavelengths were measured with a wavelength meter (WS6-600 HighFinesse) and the Ti:sapphire laser wavelengths were measured by a wavelength meter (WSU-2 HighFinesse) calibrated by measuring a reference wavelength provided by a stabilized diode laser (DLC DL PRO 780, Toptica).

\section{Collinear and anti-collinear excitation}

For the initial peak searching, a zero-degree mirror at the end of the beam line was used to reflect the laser light anti-collinearly with respect to the travelling direction of the RaF bunch. Thus, each scanning laser covered two different wavenumber regions in the molecular rest frame, owing to the Doppler shift present for the fast RaF molecules. For a molecule travelling at velocity $v$, the laser wavenumber in the laboratory frame, $\tilde{v}_{0}$, is related to the wavenumber in the molecule rest frame, $\tilde{v}$, by the expression $\tilde{v}=\frac{1+\beta \cos \theta}{\sqrt{1-\beta^{2}}} \tilde{v}_{0}$, with $\beta=v / c(c$, speed of light in vacuum) and where $\theta$ is the angle between the direction of the laser beam and the velocity of the molecule. For RaF molecules at 39,998(1) eV $\left(v \approx 0.18 \mathrm{~m} \mathrm{ss}^{-1}\right)$, a difference of $15.7 \mathrm{~cm}^{-1}$ is obtained between the laser pulse sent out collinearly $(\cos \theta=1)$ and anti-collinearly $(\cos \theta=-1)$ with respect to the direction of the velocity of the molecule.

\section{Spectroscopic analysis}

The peaks in the different spectra were identified by rebinning the spectra using coarse bin sizes with values up to $1 \mathrm{~cm}^{-1}$. Only groups of data points that were consistently observed with a 5-sigma significance above background were considered as candidates for transitions. The vibrational transitions in Fig. 2 show asymmetric line profiles with a maximum located towards higher wavenumbers. The band centres cannot be determined directly from the measured line profiles, and so we used the wavenumber positions of the maxima in our data analysis. Extended Data Table 1 lists the maximum peak positions and estimated uncertainties are given in parentheses. The wavenumber difference, $\Delta \tilde{v}$, of vibrational levels in the electronic ${ }^{2} \Sigma^{+}$ground state and in the ${ }^{2} \Pi_{1 / 2}$ excited state were derived from combination differences of the recorded ${ }^{226} \mathrm{RaF}$ spectra (see Extended Data Table 1 ).

In our analysis we used vibrational energy terms $E_{v} /(h c)$ of a Morse potential according to:

$$
E_{v} /(h c)=\tilde{\omega}_{\mathrm{e}}\left(v+\frac{1}{2}\right)-\frac{\tilde{\omega}_{\mathrm{e}}^{2}}{4 \tilde{\mathcal{D}}_{\mathrm{e}}}\left(v+\frac{1}{2}\right)^{2}
$$

Energy-level differences

$$
\left(E_{v+1}-E_{v}\right) /(h c)=\tilde{\omega}_{\mathrm{e}}-\frac{\tilde{\omega}_{\mathrm{e}}^{2}}{2 \tilde{\mathcal{D}}_{\mathrm{e}}}(v+1)
$$

were used to derive the Morse potential parameters $\tilde{\omega}_{\mathrm{e}}$ and $\tilde{\mathcal{D}}_{\mathrm{e}}$ from a least-squares fit analysis. The derived energy-level differences are given in Extended Data Table 1, whereas Extended Data Table 2 contains the molecular parameters from the fit. The harmonic vibration frequencies $\tilde{\omega}_{\mathrm{e}}$ of the ${ }^{2} \Sigma^{+}$and ${ }^{2} \Pi_{1 / 2}$ states are almost identical and correspond well to the theoretical predictions with a deviation of less than $5 \%$; see Extended Data Table 2. The same holds for the estimated dissociation energy $\tilde{\mathcal{D}}_{\mathrm{e}}$, which is in better agreement with the values of ref. ${ }^{6}$, as therein also the low-energy part of the potentials was used to estimate the dissociation energy. 


\section{Article}

In the case of the two low-lying ${ }^{2} \Pi$ fine-structure levels, the observed origins $T_{0,0}$ agree well with the calculated values based on the Relativistic Correlation Consistent - Atomic Natural Orbital (RCC-ANO) basis set. From the energy difference of the fine-structure components the effective spin-orbital coupling parameter $A$ is derived. For the ${ }^{2} \Pi$ states, the experimental value of $2,068(5) \mathrm{cm}^{-1}$ is in good agreement with the calculated value. The band origins are in reasonable agreement with results from the RCC-ANO basis set calculation, if one attributes the $\Omega=3 / 2$ levels, which were computationally found to be of mixed $\Pi_{3 / 2}$ and $\Delta_{3 / 2}$ character in this order of energies. A reverse assignment also gives better agreement with experiment. Calculations of the gas-phase bond lengths, dissociation energies and additional properties of $\mathrm{RaF}$ molecules have been reported $^{8,32,35}$.

\section{Data availability}

Examples of vibronic spectra measured for RaF molecules are included as source data with this Article. All other relevant data supporting the findings of these studies are available from the corresponding author upon request.

41. Böhlen, T. T. et al. The FLUKA code: developments and challenges for high energy and medical applications. Nucl. Data Sheets 120, 211-214 (2014).

42. HSC Chemistry 9 v.9.8.1.2 (Outotec, 2018)

43. Kreim, S. et al. Recent exploits of the ISOLTRAP mass spectrometer. Nucl. Instrum Meth. B 317, 492-500 (2013).

44. Wolf, R. N. et al. Isoltrap's multi-reflection time-of-flight mass separator/spectrometer. Int. J. Mass Spectrom. 349-350, 123-133 (2013).
45. Guglielmetti, A. et al. New measurement of exotic decay of ${ }^{225} \mathrm{Ac}$ by ${ }^{14} \mathrm{C}$ emission. Eur. Phys. J. A 12, 383-386 (2001).

Acknowledgements This work was supported by the ERC Consolidator grant no. 648381 (FNPMLS); Deutsche Forschungsgemeinschaft (DFG, German Research Foundation) project no. 328961117 - SFB 1319 ELCH; STFC grants ST/L005794/1, ST/L005786/1 and ST/PO04423/1 and Ernest Rutherford grant no. ST/LOO2868/1; projects from FWO-Vlaanderen, GOA 15/010 from KU Leuven and BriX IAP research programme no. P7/12; European Union grant agreement no. 654002 (ENSAR2); the Russian Science Foundation under grant no. 18-12-00227; and BMBF grants 05P15HGCIA and 05P18HGCIA. We thank J. P. Ramos, J. Ballof and T. Stora for their support in the production of RaF molecules. A.J.B. was suppported by the Henry W. Kendall (1955) Fellowship. We would also like to thank the ISOLDE technical group for their support and assistance. We thank D. Budker for comments and suggestions as well as A. Petrov for discussions on $\Delta$ states. R.B. acknowledges I. Tietje for early discussions on various experiments at CERN and thanks A. Welker for sharing knowledge on isotope production and separation as well as for initial discussions on the RaF studies. R.B. acknowledges discussions with K. Gaul on molecular properties and with D. Andrae on finite nuclear size effects. R.B. and T.A.I. acknowledge S. Hoekstra and L. Willmann for early discussions on the production of RaF. T.A.I. thanks A. Zaitsevskii for discussions on the coupled-cluster method.

Author contributions R.F.G.R. led the experiments and R.B. led the theoretical support for this work. R.F.G.R., R.B., C.L.B., M.L.B., K.C., B.S.C., K.T.F., R.P.d.G., S.F., F.P.G., Á.K., H.A.P., C.M.R., S.R., A.R.V., F.W. and S.G.W. performed the experiment. R.F.G.R., R.B., A.A.B., A.J.B. and T.F.G. performed the data analysis. R.F.G.R. prepared the figures. R.B. and T.A.I. performed theoretical predictions that motivated the experimental proposal and analysis of the results. R.F.G.R. and R.B. prepared the initial draft of the manuscript with input from A.A.B., A.J.B., K.T.F., T.F.G., T.A.I., G.N. and S.G.W. All authors discussed the results and contributed to the manuscript at different stages.

Competing interests The authors declare no competing interests.

Additional information

Correspondence and requests for materials should be addressed to R.G.R. or R.B.

Peer review information Nature thanks Michael Patzschke and the other, anonymous, reviewer(s) for their contribution to the peer review of this work.

Reprints and permissions information is available at http://www.nature.com/reprints. 


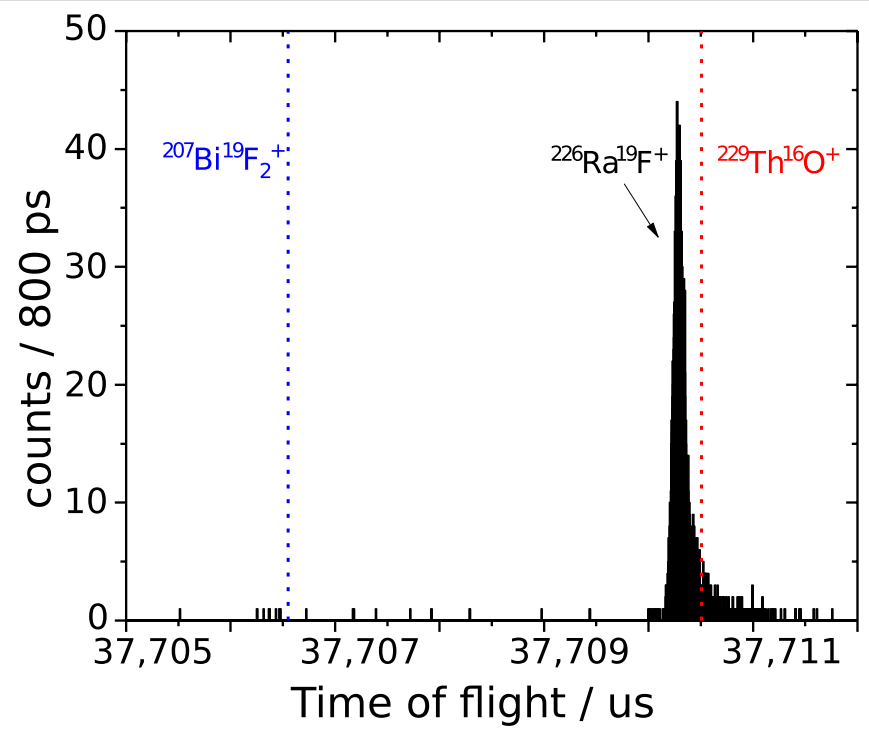

Extended Data Fig. $1 \mid$ Time-of-flight spectrum measured at mass $A=245$.

The time-of-flight spectrum of the ${ }^{226} \mathrm{RaF}^{+}(A=245)$ beam as delivered from ISOLDE after 1,000 revolutions in the multi-reflection time-of-flight mass spectrometer. A mass resolving power of $1.7 \times 10^{5}$ was achieved, which allowed the isobaric beam composition to be analysed. Only ${ }^{226} \mathrm{Ra}^{19} \mathrm{~F}^{+}$ions were detected. The positions of the most probable accompanying ions are highlighted by dotted vertical lines. 


\section{Article}

Extended Data Table 1 $\mid{ }^{226}$ RaF vibrational transitions in the electronic $X^{2} \Sigma^{+}$ground state and the $A^{2} \Pi_{1 / 2}$ excited state derived from combination differences

\begin{tabular}{cll}
\hline \hline$v^{\prime} \leftarrow v^{\prime \prime}$ & ${ }^{2} \Sigma^{+} \Delta \tilde{\nu} / \mathrm{cm}^{-1}$ & ${ }^{2} \Pi_{1 / 2} \Delta \tilde{\nu} / \mathrm{cm}^{-1}$ \\
\hline $1-0$ & $438.4(7)$ & $432.2(7)$ \\
$2-1$ & $435.0(7)$ & $428.9(7)$ \\
$2-1$ & $435.4(11)$ & $429.3(11)$ \\
$3-2$ & $431.6(11)$ & $425.6(7)$ \\
$4-3$ & $428.4(14)$ & $422.2(14)$ \\
$5-4$ & $\cdots$ & $419.2(14)$ \\
\hline \hline
\end{tabular}

Combined statistical and systematic uncertainties are given in parentheses. 
Extended Data Table 2 | Molecular parameters of RaF from vibrational analysis of the electronic ground state $\left(X^{2} \Sigma^{+}\right)$and excited states $\left(A^{2} \Pi, B^{2} \Delta\right.$ and $\left.C^{2} \Sigma^{+}\right)$

\begin{tabular}{|c|c|c|c|c|c|}
\hline & $\tilde{\omega}_{e} / \mathrm{cm}^{-1}$ & $\tilde{T}_{e} / 10^{4} \mathrm{~cm}^{-1}$ & $A / 10^{3} \mathrm{~cm}^{-1}$ & $\tilde{\mathcal{D}}_{e} / 10^{4} \mathrm{~cm}^{-1}$ & Ref \\
\hline \multirow[t]{3}{*}{$X^{2} \Sigma^{+}$} & $441.8(1)$ & & & $2.92(5)$ & this work \\
\hline & 432 & & & 3.21 & {$[43]$, theo. $^{\mathrm{a}}$} \\
\hline & 431 & & & 4.26 & [43], theo. ${ }^{\mathrm{b}}$ \\
\hline \multirow{3}{*}{$A^{2} \Pi_{1 / 2} /{ }^{2} \Pi_{3 / 2}$} & $435.5(1) / 419.1(2)$ & $1.32878(1) / 1.53554(3)$ & $2.0676(36)$ & $2.90(3) /-$ & this work \\
\hline & $428 / 410$ & $1.40 / 1.60$ & 2.0 & $3.13 /-$ & {$[43]$, theo. ${ }^{\mathrm{a}}$} \\
\hline & $428 / 415$ & $1.33 / 1.50$ & 1.7 & & [43], theo. ${ }^{\mathrm{b}}$ \\
\hline \multirow[t]{3}{*}{$B^{2} \Delta_{3 / 2} /{ }^{2} \Delta_{5 / 2}$} & $431.9(2) /-$ & $1.51477(2) /-$ & $-/-$ & $2.83(11) /-$ & this work \\
\hline & $432 / 419$ & $1.64 / 1.71$ & 0.4 & & {$[43]$, theo. $^{\mathrm{a}}$} \\
\hline & $431 / 423$ & $1.54 / 1.58$ & 0.2 & & [43], theo. ${ }^{\mathrm{b}}$ \\
\hline$C^{2} \Sigma$ & $430.9(2)$ & $1.61806(1)$ & & $2.78(9)$ & this work \\
\hline
\end{tabular}

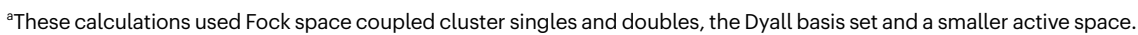

${ }^{\text {b}}$ These calculations used Fock space coupled cluster singles and doubles, the RCC-ANO basis set and a larger active space.

Experimental results are compared with theoretical predictions ${ }^{43}$, theo.

For a direct comparison, theoretical values given for $\widetilde{\omega}_{\mathrm{e}}$ should be scaled by $\sqrt{m_{\mathrm{p}} / u} \approx 1.0036$ to account for the atomic mass constant instead of the proton mass, $m_{\mathrm{p}}$, in atomic mass units $(u)$. 